\title{
Post-editing for Professional Translators: Cheer or Fear?
}

\author{
Sergi Alvarez Vidal \\ Antoni Oliver \\ Toni Badia
}

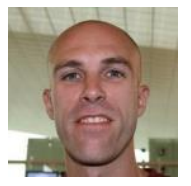

Sergi Alvarez Vidal Universitat Pompeu Fabra salvarezvid@uoc.edu ORCID: 0000-0003-4361-1250

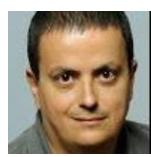

Antoni Oliver Universitat Oberta de Catalunya aoliverg@uoc.edu ORCID: 0000-0001-8399-3770

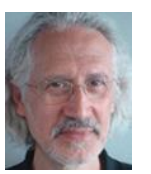

Toni Badia

Universitat Pompeu Fabra toni.badia@upf.edu ORCID: 0000-0002-9429-5940

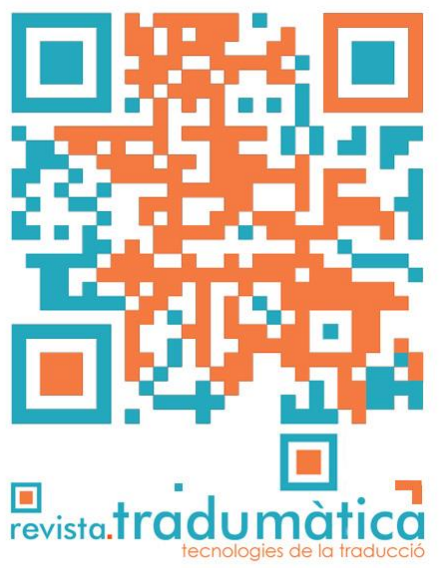

Abstract

Currently, post-editing of machine translation (MT) has been introduced as a regular practice in the translation workflow, especially since the good results in quality obtained by neural MT (NMT). This fact is linked to the efforts LSPs and customers have done to reduce costs due to the recent global crisis and the increasing globalization, which has had a negative impact on translators' revenues and on their working practices. In this context, post-editing is often perceived with a negative bias by translators. We study attitudes of translators post-editing for the first time and relate them to their productivity rates. We also compare the results with a survey answered by professional post-editors assessing their perception of the task in the current marketplace.

Keywords: machine translation, post-editing, NMT, translation workflow, translators' perceptions

Resum

Actualment, la postedició de traducció automàtica (TA) és considerada una pràctica habitual en el flux de treball de la traducció, sobretot per la bona qualitat que s'obté amb la traducció automàtica neuronal (TAN). Aquest fet està assocat als esforços que han fet els proveïdors de serveis lingüístics i els clients per reduir els costos a causa de la crisi mundial dels darrers temps i la creixent globalització, que ha tingut un impacte negatiu sobre els ingressos dels traductors i sobre les seves pràctiques professionals. En aquest cotext, els traductors acostumen a percebre la postedició amb un biaix negatiu. En aquest article es presenta un dels primers estudis sobre les actituds dels traductors envers la postedició i es relacionen amb les seves taxes de productivitat. També acarem els resultats amb una enquesta contestada per posteditors professionals que avaluen la seva percepció de la tasca en el mercat actual.

Paraules clau: traducció automàtica, postedició,

TAN, flux de treball de traducció, percepció dels traductors 
Actualmente, la posedición de traducción automática (TA) se considera una práctica habitual en el flujo de trabajo de traducción, sobre todo por la buena calidad que se obtiene con la traducción automática neuronal (TAN). Este hecho está asociado a los esfuerzos que han hecho los proveedores de servicios lingüísticos y los clientes para reducir los costos debido a la reciente crisis mundial y a la creciente globalización, que ha tenido un impacto negativo en los ingresos de los traductores y en sus prácticas profesionales. En este contexto, los traductores suelen percibir la posedición con un sesgo negativo. En este artículo se presenta uno de los primeros estudios estudio sobre las actitudes de los traductores ante la posedición y se relacionan con sus tasas de productividad. También cotejamos los resultados con una encuesta contestada por poseditores profesionales que evalúan su percepción de la tarea en el mercado actual.

Palabras clave: traducción automática, posedición, TAN, flujo de trabajo de traducción, percepción de los traductores

\section{Introduction}

Machine translation (MT) has been used as part of the translation workflow since the 1980s. However, in the last years, the good results obtained by neural machine translation (NMT) in terms of quality (Bojar et al. 2018) have attracted the attention of both research and industry. These promising results have driven a technological shift from (phrase-based) statistical machine translation (SMT) to neural machine translation (NMT) in many translation industry scenarios. As part of this change, machine translation post-editing (PE) has increased its presence in the translation workflow and half of the LSPs offer it as a service (Lommel and Depalma 2016).

In the last decade, the translation marketplace has suffered important changes that have also affected the translation profession. Both the rapid globalization propelled by the neoliberal policies and the recent global economic crisis have increased the effort from LSPs and customers to reduce costs in the translation workflow, which has had an important impact both on translators' revenues and working practices (Moorkens 2017). Furthermore, the increased technologisation means that many translators need to include different working routines into their profession, which in some cases may limit the scope of their work (Vieira and Alonso 2018).

The current study has two objectives: firstly, to assess the attitude of translators who are post-editing MT output for the first time before and after the task and to relate it to productivity rates in an industrial scenario. Secondly, to compare the results with the perceptions of current professional post-editors.

To achieve our objectives, we set four professional translators to the task of postediting and translating from scratch from Spanish into English general domain texts. Translators answered questions regarding their perceptions and attitude before and after post-editing, and we recorded the number of keystrokes and the time spent while performing the tasks. Additionally, 50 participants answered an online survey we conducted addressed to professional post-editors regarding their perception of the job.

The paper is structured as follows. First, we review previous research on post-editing and translators' perceptions regarding machine translation. Then, we specify the 
methodology followed in this paper. In the next section, we detail the post-editing task carried out by translators. It includes the description of the LSP, the questionnaires the four translators answered before and after post-editing for the first time and the report on productivity, measured as technical and temporal effort. We also study the results from an online survey answered by 50 post-editors regarding their current perception of post-editing. Finally, we discuss the results.

\section{Related Work}

Post-editors "edit, modify and/or correct pre-translated text that has been processed by an MT system from a source language into (a) target language(s)" (Allen 2003, 296). In the best scenario, MT output only requires some small modifications, but sometimes post-editors delete the remaining segments and translate everything from scratch if they consider it will take them less time (Parra-Escartín and Acedillo 2015).

In the last years, a lot of empirical investigations have been conducted to analyze post-editing because it is increasing its presence in the translation workflow (Lommel and Depalma 2016). This is due to the good results obtained by NMT in terms of quality (Bojar et al. 2018) and the need to reduce costs (Guerberof 2009a; Sosoni and Rogers 2013) and shorten time cycles. Results consistently show that post-editing is faster than translating from scratch (O'Brien 2005; Plitt and Masselot 2010), although for general language texts some studies see no significant improvement in speed (Screen 2017). However, post-editing productivity means "not only the ratio of quantity and quality to time but also the cognitive effort expended; and the higher the effort, the lower the productivity" (O'Brien 2011, 198).

There are several works studying post-editing effort (Specia 2011, Koponen 2016, Jia et al. 2019), all of which use the division established by Krings (2001): temporal effort (time spent post-editing), technical effort (number of edits, often measured using keystroke analysis), and cognitive effort (usually measured with eye-tracking or thinkaloud protocols). Research shows cognitive effort correlates with technical and temporal PE effort (Moorkens et al. 2015). Additionally, records of gaze data reveal that the reading time of the source text and target text is very different in post-editing compared to from-scratch translation (Carl et al. 2015; Daems et al. 2017).

More than two decades ago, translation memories (TMs) were first introduced in the translation arena and have since been adopted by the majority of translators (Christensen and Schjoldager 2016; Doherty 2016). Currently, very few translators ignore tools such as term banks, translation memory systems and quality checkers in the daily translation tasks. However, studies show that translators still regard the use of technology mainly as a threat (Katan 2011). LeBlanc (2013) reported on translators' perception of TMs and described the main advantages and disadvantages. Although translators admitted it helped increase productivity and reduced repetitive work, their main concern was that it was a barrier for creativity and made translators increasingly passive and lazy. 
Regarding the use of MT, research shows that translators perceive they are less productive post-editing, even when a quantitative analysis shows otherwise (Gaspari et al. 2014). They consider MT output to be tedious to post-edit (Moorkens and O'Brien 2017) and they prefer to translate from scratch even if this has a negative impact on productivity (Teixeira 2014).

Guerberof (2013) surveyed the perception of MT post-editing among current posteditors. The majority of the 27 respondents were translators already familiar with postediting who showed mixed answers. There wasn't a clear rejection to use MT and they were mainly satisfied with their jobs as post-editors. However, Läubli and OrregoCarmona (2017) analyzed posts on social media as a way to understand how translators felt about MT. They showed a negative general perception and a disconnection between the research and the translation community.

Cadwell et al. (2016) interviewed translators working at the European Commission's Directorate-General for Translation (DGT) to better understand the factors involved in the translators' adoption and non-adoption of MT during their translation tasks. They had a broadly positive attitude to MT because they believed (a) it increased speed and productivity, (b) the MT output had good quality, (c) it served as inspiration and (d) reduced typing or clicking. However, the main reasons not to use MT were (a) the perceived poor quality of MT output, (b) the negative influence it had on the translator's abilities, (c) the fear it awakened among them and (d) the extra attention needed from the translators when post-editing. A follow-up study with translation companies (Cadwell et al. 2018) highlighted mainly the same concerns, but translators also argued they were worried about the fairness of monetary compensation for postediting.

\section{Methodology}

Our aim was to study translators' perception when post-editing for the first time and compare the results to their productivity measured as technical and temporal effort. To this end, we worked with an LSP called Incyta. Four experienced translators without previous post-editing experience who usually collaborated with the LSP participated in the experiment translating from Spanish into English. First of all, translators answered a short questionnaire before post-editing to assess their attitudes towards the task. Then, we selected three general domain documents with similar terminology and type-token ratio. They translated from scratch a document of 2437 words. Then, they post-edited two documents of 2189 and 1920 words, respectively, that had been translated using DeepL. ${ }^{1}$ In both tasks, they were asked to produce printable-quality translations.

Instead of trying to reproduce the working conditions of translators, which could vary greatly among individuals, we used PET (Aziz et al 2012), a simple standalone tool that allows post-editing of MT output and translation from scratch and records information of the post-editing effort (time and keystrokes) at sentence-level. A week

1 https://www.deepl.com/translator 
before beginning the task, we sent them the tool and delivered detailed information on its use, together with a short text they could use to test it. We used this testing period to answer questions and solve usability issues so that all translators could have a fair knowledge of the tool before beginning the task.

Once the task was finished, translators answered another questionnaire with questions about their perception of the task and, in some cases, they were asked to answer follow-up questions by mail to clarify some of the answers.

Additionally, we prepared a larger survey targeting experienced post-editors to study their perception of the task and to compare it with the opinions expressed by translators post-editing for the first time. For the survey, we used the web-based SurveyMonkey platform because it allows to create online surveys that can be easily distributed and also allows to analyze and summarize all data collected in different formats. We published a 33-question survey targeted exclusively to translators who had already worked as post-editors. We tested the wording of the questions in a pilot study with two professional translators to ensure that there was no ambiguity, so that all answers could provide enough data for a complete analysis. Once the survey was ready, we published it on Linkedin and sent it to three translation associations. 50 participants answered the survey.

\section{Post-editing task}

\section{Language Service Provider}

Incyta is a Barcelona-based LSP founded in 1993. It is also the provider of Lucy Software ${ }^{2}$, a commercial rule-based machine translation engine. It has previous experience in post-editing and it is currently working with the Spanish-Catalan, SpanishGalician and Spanish-Portuguese language combinations, mainly to translate news on a daily basis for newspapers. To post-edit these language combinations, they use their own commercial MT software.

The company was interested in introducing post-editing for some new language combinations because of the increase in the demand and it was planning to implement Spanish-English post-editing for some news workflows in 2021. After the quality assessment it conducted internally, for this new language combination it was going to use a NMT engine. The translators with whom the company usually worked were quite skeptical about the quality of the MT output and the economic repercussions in their earnings and weren't willing to begin post-editing. On the other hand, the LSP wanted to continue the current collaboration with these translators due to the good quality of their work. For post-editing tasks, the company envisaged to pay translators $70 \%$ of their current rate based on their previous experience working with closely-related languages, although they were paid their regular rate for this experiment.

2 https://www.lucysoftware.com/ 


\section{Perceptions before Post-editing}

We sent the four translators taking part in the experiment a questionnaire before they began the task so that we could know their current use of technology and MT and their attitude towards post-editing. These were the questions:

Q1. How many years of experience as a professional translator do you have?

Q2. How long have you worked with this LSP?

Q3. Have you worked before correcting translation outputs?

Q4. Do you use computer-assisted translation (CAT) tools?

Q5. Are you a regular consumer of machine translation?

Q6. How do you feel about post-editing machine translation output?

Q7. What are your expectations regarding post-editing?

Q8. What do you think the MT quality is going to be?

Q9. What do you think your global experience post-editing is going to be?

Q10. To which of these tasks do you think it will be similar? (Possible answers: A. Reviewing human translations; B. Translating with fuzzy matches; C. Translating from scratch).

The four professional translators (T1, T2, T3 and T4) who carried out the task had extensive experience translating (12,16, 15 and 18 years, respectively) (Q1) and had worked more than three years with this LSP translating from Spanish into English (Q2). T1 and T2 had never used CAT tools before, while T3 and T4 used them only sometimes for certain specific projects (Q4). We also asked them about their previous experience correcting human outputs, as research has shown translators often relate it to post-editing (Guerberof 2013). T1 and T4 had done corrections of human translations before, but T2 and T3 did only translations (Q3). Although none of them were regular consumers of MT in their daily lives, they believed the general quality of MT had improved considerably in recent years (Q5).

Regarding their attitude towards post-editing (Q6), none of the translators were looking forward to it. They recognised MT was "getting better, but still cannot compare to a (decent) human translation" (T2). T4 showed concerns MT will take over the industry and T3 thought it would take as much time as translating from scratch and "can influence my own translation."

When asked about their expectations (Q7), T1 thought it would be like reviewing translations by non-natives, where "sentence structure and context acquire special importance". T2 thought it was not going to be an enjoyable job and she also had "ethical conflicts with my profession disappearing and only becoming post-editing, which is more poorly paid and frankly less fun and creative". Only T3 highlighted the quality of the MT output as a key factor. If the quality is good, it will be a "positive experience". 
Q8 and Q9 offered the participants a Likert scale where 1 was "Very bad" and 5 was "Excellent". The majority of the translators thought the quality of the MT output was going to be very good (4), but their experience post-editing was only going to be good (3). In the last question (Q9), from the three options offered as answers, T1 and T3 thought post-editing would be similar to reviewing human translations and T2 and T4 believed it would be like translating with fuzzy matches. None of them believed post-editing would be similar to translating from scratch.

Although their general attitudes regarding post-editing were mainly negative, they didn't think the post-editing experience was going to be bad. In fact, some of the fears expressed were related to rates and other market practices.

\section{Perceptions after Post-editing}

Once they had finished post-editing and translating from scratch, we sent them another questionnaire to collect information on their perceptions, mainly to understand what the main difficulties had been and if their opinions had changed after carrying out the task.

We asked them the following questions:

Q1. Grade the global post-editing experience

Q2. Would you be willing to post-edit on a regular basis?

Q3. What did you like best about post-editing?

Q4. What did you like less about post-editing?

Q5. Do you think following some training would improve your productivity postediting?

Q6. Do you think having more information about the MT engine would improve your productivity post-editing?

Q7. What is your assessment of the MT quality?

Q8. What were the main errors it produced?

Q9. Do you think post-editing is similar to revising human translations?

Q10. Did you find some errors difficult to spot?

Q11. Do you think you had a higher productivity than translating from scratch?

Q.12 Do you think the final translation had the same quality?

Q13. Are you as satisfied with the result as if it had been translated from scratch?

For the first questions (Q1), translators were offered again a Likert scale where 1 was "Very bad" and 5 was "Excellent". T1 and T3 thought the experience had been good (3), T2 thought it had been bad (2) and T4 qualified it as excellent (5). Except T2, who was quite disappointed with the experience, the other three translators would be willing to post-edit in a regular basis (Q2), but only if the "rate was right". T1 stressed "there is nothing enjoyable (to me) about post-editing, whereas translating is 
enjoyable," even though they recognised the improved quality of the MT output. As positive elements (Q3) they thought post-editing saved them time typing and they didn't have to correct basic mistakes. On the downsides (Q4), they thought it "constrained creativity when reformulating sentences" and it was "total roteness" because of the lack of creativity. These opinions coincide mostly with the ones expressed in previous studies regarding the adoption of MT by translators (Cadwell et al. 2016).

Three of the translators believed that a proper training would improve their performance post-editing (Q5) and two of them thought it would be positive to have information on the MT engine (Q6). Regarding the MT quality (Q7), the mean rating was 3.75 out of 5 , even though they found some important errors (Q8) while postediting. T1 and T3 highlighted the high number of grammatical errors, while T2 thought the main errors were "too-literal translation of the sentences" and missing nuances. T4 also detected some inconsistencies (pliego had been translated both as document and specifications, sobre had been translated as about and envelope) and some words that had been badly translated (unión temporal had been translated as temporary union instead of joint venture; garantía had been translated as security instead of bid bond).

All four translators agreed that post-editing was not similar to revising human translations (Q9) because the errors were of "a different nature". They also explained (Q10) that some errors were hard to spot because there was a lack of uniformity. There were not usual errors, such as "typos and spelling mistakes and I had to pay special attention to the actual translation". This perception is in line with the results of research carried out comparing error annotations of statistical MT and neural MT (Klubicka et al. 2018).

All four translators agreed productivity was higher when post-editing (Q11) but that this was not the only important factor. They stated a lower degree of satisfaction ("I become a 5th-grade teacher correcting essays, and that is not the profession I signed up for!"). They also agreed their final product was of similar quality as if they had translated it from scratch (Q12). They were proud of the results (Q13) after all the corrections had been introduced.

In general, their experience was better than they had expected. They found the MT quality to be good enough, although mistakes were sometimes difficult to spot.

\section{Productivity results}

We analyzed the technical and temporal effort collected during the translation with PET to calculate the productivity differences between post-editing and translating from scratch. As it can be seen in Table 1, although there is a great variability among translators, the mean shows there is an increase of $53.14 \%$ in productivity in words per hour if we compare the translation from scratch and the post-editing task. This increase ranges fom $33.29 \%$ in the case of $\mathrm{T} 1$ to a $81.01 \%$ in the case of T3. If we consider exclusively the productivity figures, the rate reduction of $30 \%$ suggested by the LSP could be considered in line with these results. 
Regarding the technical effort, calculated in keystrokes per word, Table 2 shows there is a reduction of $71.69 \%$. T1 shows the lower reduction with $24.38 \%$ while T2 shows the highest decrease with $81.84 \%$. As post-editors have to correct the MT output, there is much less typing involved.

\begin{tabular}{|c|c|c|c|c|c|}
\hline & T1 & T2 & T3 & T4 & Mean \\
\hline $\begin{array}{c}\text { From } \\
\text { scratch }\end{array}$ & 935.51 & 1994.36 & 486.32 & 560.59 & 994.19 \\
\hline PE & 1246.92 & 3209.27 & 880.27 & 753.62 & 1522.54 \\
\hline
\end{tabular}

Table 1. Temporal effort in words per hour.

\begin{tabular}{|c|c|c|c|c|c|}
\hline & T1 & T2 & T3 & T4 & Mean \\
\hline $\begin{array}{c}\text { From } \\
\text { scratch }\end{array}$ & 4.47 & 6.83 & 24.47 & 17.06 & 13.21 \\
\hline PE & 3.38 & 1.24 & 4.66 & 5.70 & 3.74 \\
\hline
\end{tabular}

Table 2. Technical effort in characters per word.

\section{Survey for post-editors}

In our study, translators who post-edited for the first time showed in general a negative perception of the task even though there was an increase in their productivity. We prepared a larger survey to ask translators with experience in post-editing what their opinions were. We wanted to know if the knowledge and expertise gained through training and experience had affected their current post-editing practices and also what their general working conditions were in relation to rates, professional satisfaction and their working environment. Even though only 50 post-editors participated in the survey, the answers can be used to obtain a fair picture of the current perception of posteditors regarding their job.

In the first question (Q1), we asked them to introduce a user ID in order to identify them. In the second question (Q2), we asked participants about their working language pairs. Most of them worked with European languages such as English, German, Spanish, Italian and Portuguese, which are common language combinations in MT engines. Then, we asked them if they had followed studies in translation (Q3) and if they had any training in post-editing (Q4). The majority of the participants had completed translation studies at university (60\%). However, only some of them had followed some training or instructions on post-editing (42\%). 
Have you completed any studies in translation?

Answered: 50 Skipped:0

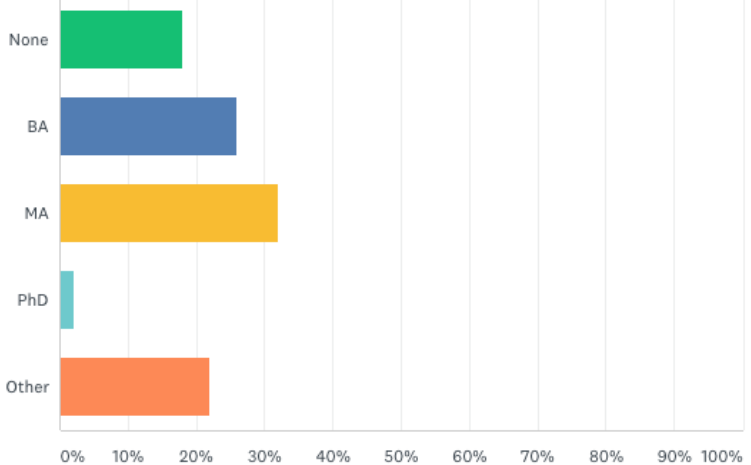

ANSWER CHOICES

- None

- BA

- MA

PhD

Other

TOTAL

\begin{tabular}{|l|c|}
\hline RESPONSES & - \\
\hline $18.00 \%$ & 9 \\
\hline $26.00 \%$ & 13 \\
\hline $32.00 \%$ & 16 \\
\hline $2.00 \%$ & 1 \\
\hline $22.00 \%$ & 11 \\
\hline & 50 \\
\hline
\end{tabular}

Figure 1. Answers regarding studies in translation (Q2).

Have you followed any training in post-editing?

Answered: 50 Skipped: 0

Yes

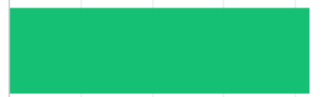

No

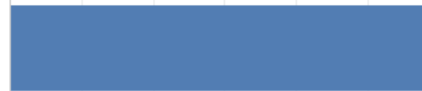

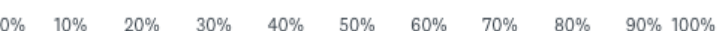

\begin{tabular}{l|cc} 
ANSWER CHOICES & RESPONSES & - \\
\hline YES & $42.00 \%$ & 21 \\
\hline NO & $58.00 \%$ & 29 \\
\hline TOTAL & & 50
\end{tabular}

Figure 2. Answers regarding training in post-editing (Q3).

To all of those who had received some sort of training, we asked them to state which one and give their opinion about the quality of the training (Q5). Most of them explained they were only given instructions about the post-editing process and the final quality required (52\%). Some of them received training by the LSP they were working 
for (17\%) when they first began post-editing, and the remaining translators (31\%) followed a more formal course, such as the ones developed by SDL or TAUS. All participants who followed these courses found them helpful. All respondents who had not followed any training agreed it would have been a great help when they began post-editing (Q6).

Currently many university translation programmes have acknowledged the need to go beyond the teaching of translation memories (TM) in technology modules and to include post-editing courses across the curriculum because this task requires a specific set of skills (O’Brien 2012; Kenny and Doherty 2014; Mellinger 2017), which can be grouped in three main competences: core, linguistic and instrumental (Rico and Torrejón 2012). Some authors have also highlighted the importance of using tailored post-editing guidelines that express without ambiguity the goals of the task to be performed (Flanagan and Christensen 2014; Hu and Cadwell 2016).

Then, we asked about the amount of experience they had translating (Q7) and post-editing (Q8). As we can see in Figures 3 and 4, 43 participants (86\%) had more than three years of experience translating (86\%). However, only 17 respondents (34\%) had a similar amount of experience post-editing, while the majority of them (50\%) had been post-editing for only between one and three years. This could be in part due to the recent increase in the demand of post-editing in the market (Lommel and Depalma 2016), as it reduces costs and increases productivity (Plitt and Masselot 2010). Even so, when inquired about the percentage that post-editing represented in their whole workload (Q9), for most of them (70\%) it was less than 20\%. And only $44 \%$ of the respondents stated their post-editing workload had increased (on average a 20\%) in the last few years (Q10).

How many years of experience do you have as a translator?

Answered: 50 Skipped: 0

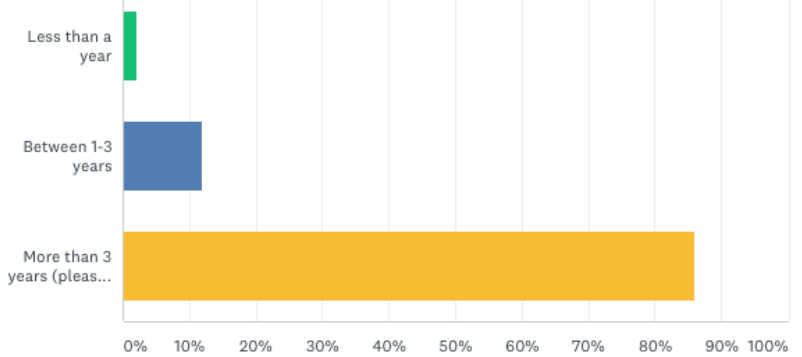

\begin{tabular}{|c|c|c|c|}
\hline ANSWER CHOICES & - & RESPONSES & - \\
\hline Less than a year & & $2.00 \%$ & 1 \\
\hline Between 1-3 years & & $12.00 \%$ & 6 \\
\hline More than 3 years (please specify) & Responses & $86.00 \%$ & 43 \\
\hline TOTAL & & & 50 \\
\hline
\end{tabular}

Figure 3. Answers regarding their experience as translators (Q7). 
How many years of experience do you have as a post-editor?

Answered: 50 Skipped: 0

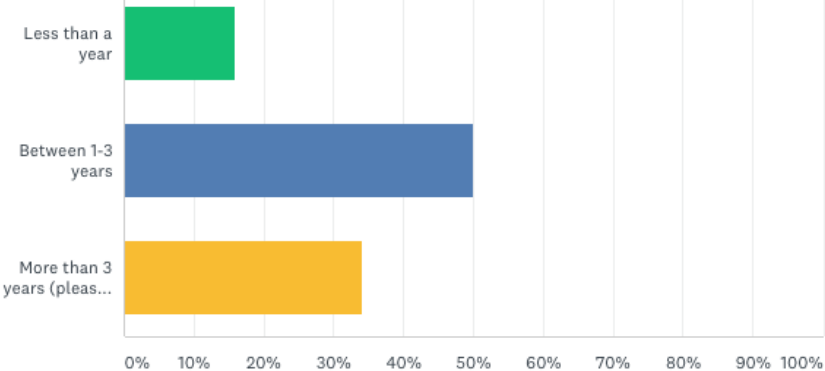

\begin{tabular}{|c|c|c|c|}
\hline ANSWER CHOICES & . & RESPONSES & . \\
\hline Less than a year & & $16.00 \%$ & 8 \\
\hline Between 1-3 years & & $50.00 \%$ & 25 \\
\hline More than 3 years (please specify) & Responses & $34.00 \%$ & 17 \\
\hline TOTAL & & & 50 \\
\hline
\end{tabular}

Figure 4. Answers regarding their experience as post-editors (Q8).

We also asked about the type of texts they post-edited (Q11), which were usually technical or medical, domains in which post-editing has traditionally achieved better results (Aymerich 2005; Kirchoff 2011). Very few translators (5\%) post-edited general domain documents.

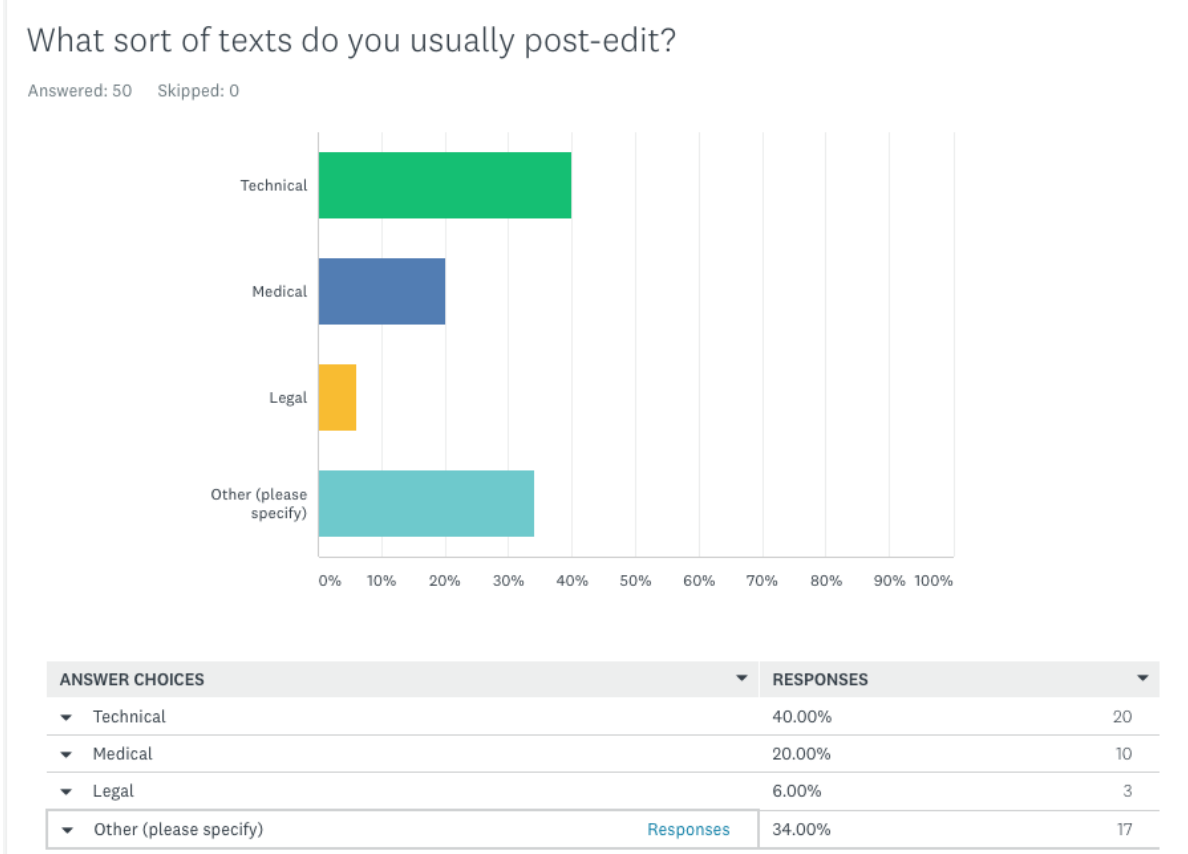

Figure 5. Types of documents they post-edited (Q11)

When asked to select among different statements which described with more accuracy their progress post-editing along time, $70 \%$ of the participants agreed that experience had led them to better detect MT errors (Q12) and post-edit faster (Q13), which is in line with the results obtained in previous studies relating experience with 
higher efficiency (Moorkens and O'Brien 2014), even though some participants stressed the great variation of errors found in the MT outputs. However, regarding the effort it entailed in relation to translating (Q14), 50\% considered it required more effort than translating using translation memories and $22 \%$ believed it was a task that entailed more effort than revising human translations.

\begin{tabular}{|c|c|c|c|}
\hline ANSWER CHOICES & $\checkmark$ & RESPONSES & - \\
\hline I can spot MT errors better now. & & $70.00 \%$ & 35 \\
\hline I feel that there has been no change in my capacity to spot MT errors with experience. & & $22.00 \%$ & 11 \\
\hline I can spot less MT errors now because I'm used to them. & & $4.00 \%$ & 2 \\
\hline I don't know (describe your impressions) & Responses & $4.00 \%$ & 2 \\
\hline TOTAL & & & 50 \\
\hline
\end{tabular}

Figure 6. Multiple-choice question regarding MT error detection (Q12)

\begin{tabular}{|c|c|c|c|}
\hline ANSWER CHOICES & 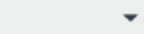 & RESPONSES & $\checkmark$ \\
\hline Experience has helped me post-edit faster. & & $70.00 \%$ & 35 \\
\hline I feel that there has been no change in my post-editing speed with experience. & & $14.00 \%$ & 7 \\
\hline I post-edit slower now. & & $2.00 \%$ & 1 \\
\hline I don't know (describe your impressions) & Responses & $14.00 \%$ & 7 \\
\hline TOTAL & & & 50 \\
\hline
\end{tabular}

Figure 7. Multiple-choice question regarding post-editing experience (Q13)

In order to obtain a better picture of their current working situation, we asked about post-editing rates. As we can see in Figure 8, participants in the survey mostly believed that at the end of a day in which they only post-edited, they earned less money (52\%) or the same amount of money (36\%) than if they had been translating (Q15). This fact can be linked to the effort LSPs and customers have done to reduce costs since the 2008 crisis, which has negatively affected translating rates (Moorkens 2017). Regarding the quality of the MT they had to post-edit (Q16), most of them thought it was acceptable though it needed many editions (44\%), or they even believed that it was of borderline quality (22\%). Another $22 \%$ of the respondents even erased the whole MT output and translated from scratch in certain segments throughout the task although they were only paid for post-editing. It is well known that the quality of the MT output is a key element in post-editing as it affects the productivity gain (Garcia 2011). 
In your opinion, how fair are post-editing rates in relation to the time spent?

Answered: 50 Skipped: 0
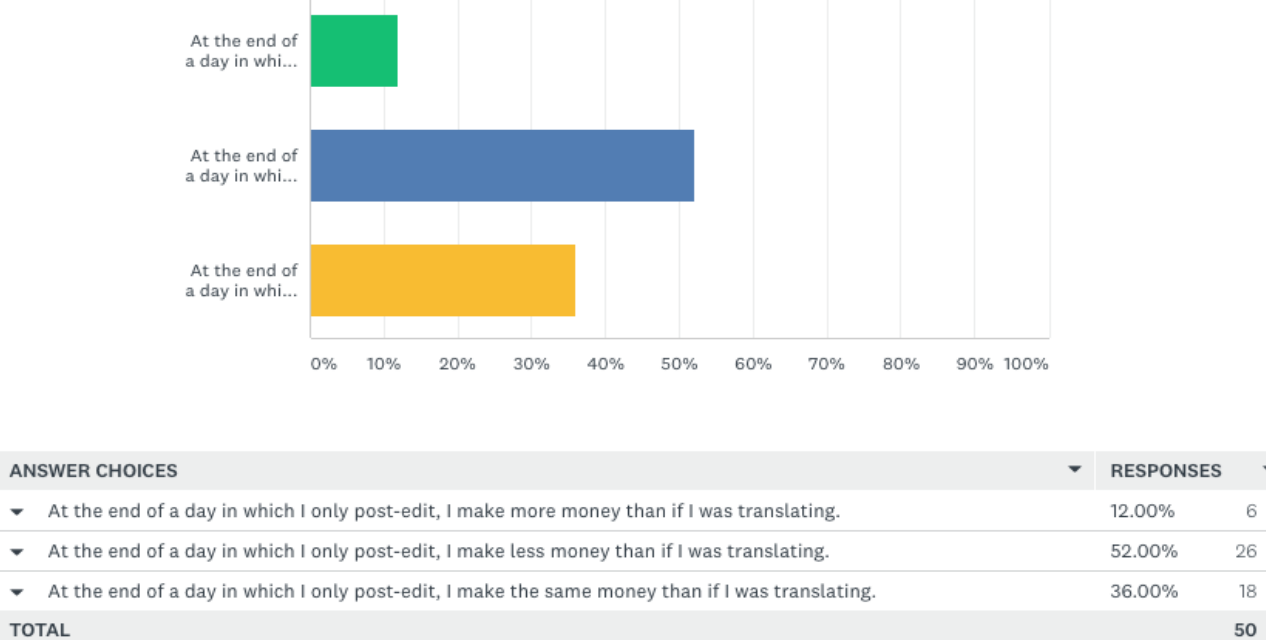

Figure 8. Correlation between post-editing rates and time spent (Q15)

The majority of participants clearly stated that post-editing rates were not currently adequate to the effort it entailed (66\%) (Q17) because it was more tiring than translating (38.78\%) and than revising human translations (14.29\%) (Q18). Regarding the estimation method to calculate the rate they were paid (Q19), nearly half of them preferred being paid by word (44\%) although $26 \%$ felt comfortable with both payment methods.

Are rates adequate to the effort required for PE? Think about what you can earn at the end of a full day of only doing PE.

Yes.

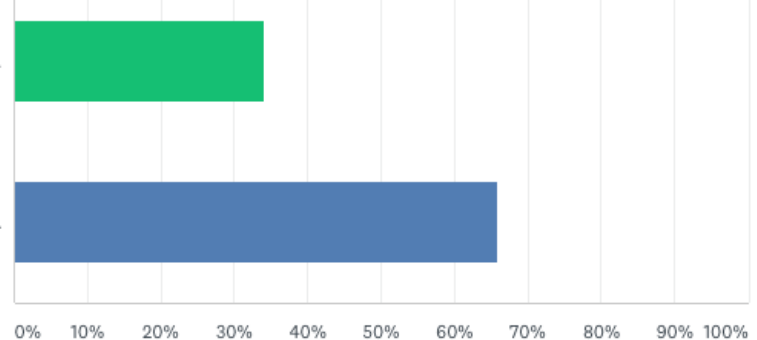

Figure 9. Correlation of effort and rates (Q17) 
How would you compare the level of tiredness after a full day post-editing?

Answered: 50 Skipped: 0

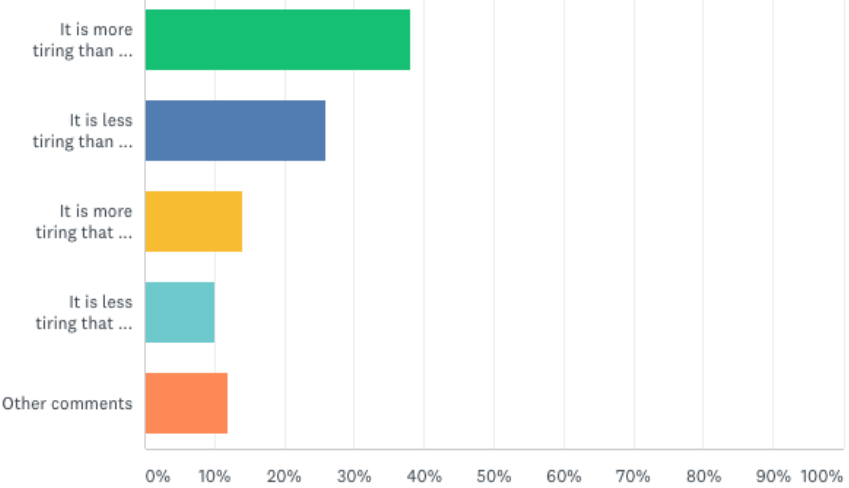

\begin{tabular}{|c|c|c|c|}
\hline ANSWER CHOICES & & RESPONSES & - \\
\hline It is more tiring than if I were translating. & & $38.00 \%$ & 19 \\
\hline It is less tiring than if I were translating. & & $26.00 \%$ & 13 \\
\hline - It is more tiring that if I were revising human translations. & & $14.00 \%$ & 7 \\
\hline It is less tiring that if I were revising human translations. & & $10.00 \%$ & 5 \\
\hline Other comments & Responses & $12.00 \%$ & 6 \\
\hline TOTAL & & & 50 \\
\hline
\end{tabular}

Figure 10. Level of tiredness implied in post-editing (Q18)

Regarding the tools they used, all translators who answered the survey used the same tool to translate (Q20) and to post-edit (Q21). They mainly used SDL Trados Studio (55\%), followed by MemoQ (16\%) (Q22). As it can be seen in Figure 9, most of them explained the tool they were currently using was the best suited for post-editing (74\%) (Q23).

Do you think the tool you are currently using is the best suited for postediting?

Answered: 50 Skipped: 0

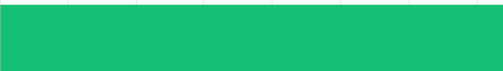

No.

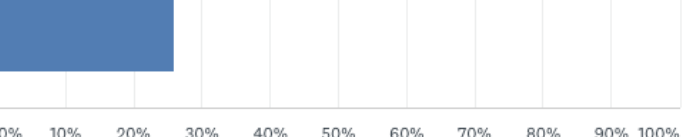

\begin{tabular}{lcc} 
ANSWER CHOICES & RESPONSES & - \\
- YES. & $74.00 \%$ & 37 \\
\hline NO. & $26.00 \%$ & 13 \\
\hline TOTAL & & $\mathbf{5 0}$
\end{tabular}

Figure 11. Suitability of current tool for post-editing (Q23).

However, Moorkens and O'Brien (2017) concluded after an extensive survey that currently post-editing was not well-supported by existing tools and there was a need to 
study new specifications for user interfaces (Uls) that better supported the post-editing task. To get the participants insight, we asked them if they would like to add any additional functionality for post-editing to the tool they were currently using (Q24). $20 \%$ of the respondents suggested that the propagation of post-editor's corrections would be very useful and could save the more repetitive and edit-intensive tasks, although three post-editors stressed the fact that errors are not always the same, especially when translating lexical elements. Another post-editor thought it would be useful to include measurements of post-editing effort while translating, instead of having to wait until the post-editing had been finished. This could be useful to give the post-editors some insight regarding their progress while post-editing.

When asked about any additional element they would incorporate to the UI (Q25), participants mainly suggested adding more shortcuts for tag insertions, providing automatic corrections and including tools to help rearrange words in a sentence.

Another important element when post-editing is the MT system used to produce the output. Post-editors who answered the survey did not usually (38\%) or never (26\%) receive any information regarding the MT system (Q26). However, this could be a useful piece of information as recent research has shown that different MT models produce different types of errors (Klubicka et al. 2018). In fact, most respondents (67\%) believed that if translators were trained on understanding how MT works they would feel more confident post-editing (Q27).

In the following questions, we enquired participants to rate their satisfaction level with the translation tasks (Q28) and post-editing tasks (Q29) giving a mark from 0 ("Very bad") to 100 ("Excellent"). For translation, the mean rating was 83, while postediting obtained a 56. Additionally, we asked them to explain the main reasons for the previous rating of translating (Q30) and post-editing (Q31). They thought translating boosted creativity and gave translators the chance to work with different text types. However, respondents showed more concerns about post-editing. They found it was more boring and repetitive. They believed that having to correct computer-generated errors tended to be tedious, as they usually "have to correct as little as possible to be profitable, so we do not aim for the best quality". One respondent even suggested that "the hardest is to remember what is genuine in the language."

Finally, we asked them if they thought their voices were heard in industrial workflows (Q32) and what suggestions would they make to improve this workflow (Q33). Except in two cases, they all thought their voices were never listened to, which correlates to previous surveys analyzing the current working conditions of professional translators (Vieira and Alonso 2018). They mainly stressed the importance of increasing post-editing rates, which were considered low, and improving the quality of the MT output. Post-editors also made reference to the tight deadlines in the current translation marketplace and the possibility of correcting formal or repetitive mistakes in the text before post-editing began. 
Translators who post-edited for the first time showed prejudices and a general negative attitude before post-editing. In part it was due to the characteristics of the task but also because of other external elements such as rates and the future of the translation profession. However, once they had finished post-editing, their opinions were not so negative and most of them would be willing to post-edit in a regular basis even though they all enjoyed more translating. The main challenges of post-editing were related to the constraints it imposes, mainly to creativity. Another important problem was the unpredictable errors in the MT output, which were sometimes difficult to spot. Regarding productivity, post-editing reduced in half the time spent by word.

Experienced post-editors also considered this task to be more repetitive, more tiring and less paid than translating from scratch. However, they highlighted post-editing productivity increases with experience and proper training. Moreover, post-editing reduces typing, which usually helps to increase productivity. In general, translators are less satisfied with post-editing than with translation from scratch.

According to the opinions of the participants, training and experience are key elements to post-editing with more confidence. There is also a need for a more fluent communication throughout the translation workflow, mainly related to information regarding the origin of the MT output. As Vieira and Alonso explain (2018), one of the main problems for translators is the lack of communication from project managers regarding what they are asked to do and how to do it. The focus of the translation workflow should be the human translator and MT should be used as a way to improve a human-centered process. Finally, as post-editors believe it is a more tiring task, one possibility would be to limit the time spent post-editing and always combine it with translation from scratch as part of the industrial workflow.

All in all, post-editing is a task that offers a new specialization for current translators. However, current market practices have to be improved and tuned in order to make it more attractive and enjoyable for professional translators.

\section{References}

Allen, Jeffrey H. (2003). Post-editing. In Harold Sommer (ed.), Computers and translation: a translator's guide, pp. 297-317. Amsterdam: John Benjamin. https://benjamins.com/catalog/btl.35.19all. https://doi.org/10.1075/btl.35.19all

Aziz, Wilker, S. Castilho, and Lucia Specia. (2012). "PET: A Tool for Post-Editing and Assessing Machine Translation." The Eighth International Conference on Language Resources and Evaluation, pp. 3983-3987.

Aymerich, Julia. (2005). "Using Machine Translation for Fast, Inexpensive, and Accurate Health Information Assimilation and Dissemination: Experiences at the Pan American Health Organization." 9th World Congress on Health Information and Libraries.

Salvador - Bahia, Brazil. Accessed May 1, 2020, http://www.icml9.org/program/track3/public/documents/Julia\%20Aymerich174140.pdf 
Bojar, Ondrej, Rajen Chatterjee, Christian Federmann, Yvette Graham, Barry Haddow, Matthias Huck, Antonio Jimeno Yepes, Philipp Koehn, Varvara Logacheva, Christof Monz, Matteo Negri, Aurelie Neveol, Mariana Neves, Martin Popel, Matt Post, Raphael Rubino, Carolina Scarton, Lucia Specia, Marco Turchi, Karin Verspoor, and Marcos Zampieri. (2018). "Findings of the 2018 Conference on Machine Translation." Proceedings of the First Conference on Machine Translation (WMT 2018). Vol. 2, pp. 98-131. https://doi.org/10.18653/v1/W18-6401

Cadwell, P., Castilho, S., O'Brien, S., and Mitchell, L. (2016). Human Factors in Machine Translation and Post-Editing among Institutional Translators. Translation Spaces, 5(2), pp. 222-243. https://doi.org/10.1075/ts.5.2.04cad

Cadwell, Patrick, Sharon O'Brien, and Carlos S. C. Teixeira. (2018). "Resistance and Accommodation: Factors for the (Non-) Adoption of Machine Translation among Professional Translators." Perspectives: Studies in Translatology 26(3), pp. 301-321. https://doi.org/10.1080/0907676X.2017.1337210

Carl, Michael, Gutermuth, Silke and Silvia Hansen-Schirra. (2015). "Post-editing Machine Translation: Efficiency, strategies and revision processes in professional translation settings." In Psycholinguistic and Cognitive Inquiries into Translation and Interpreting, edited by Aline Ferreira and John Schwieter. Amsterdam/Philadelphia: John Benjamins Publishing Company, pp. 145-174. https://doi.org/10.1075/btl.115.07car

Christensen, Tina Paulsen and Anne Schjoldager. (2016). "Computer-Aided Translation Tools-the Uptake and Use by Danish Translation Service Providers." The Journal of Specialised Translation. Vol. 25, pp. 89-105.

Daems, Joke, Vandepitte, Sonia, Hartsuiker, Robert J. and Lieve Macken. (2017). "Translation methods and experience: A comparative analysis of human translation and post-editing with students and professional translators." Meta 62, pp. 245-270. https://doi.org/10.7202/1041023ar

Doherty, Stephen. (2016). "Translations: The Impact of Translation Technologies on the Process and Product of Translation." International Journal of Communication 10, p. 23.

Flanagan, Marian and Tina Paulsen Christensen. (2014). "Testing post-editing guidelines: how translation trainees interpret them and how to tailor them for translator training purposes." The Interpreter and Translator Trainer 8 (2), pp. 257-275. https://doi.org/10.1080/1750399X.2014.936111

Fulford, Heather and Joaquin Granell- Zafra. (2005). "Translation and Technology: A Study of UK Freelance Translators." The Journal of Specialised Translation 4(4), pp. 2-7.

Gaspari, Federico, Antonio Toral, Sudip Kumar Naskar, Declan Groves, Andy Way. (2014). "Perception vs Reality: Measuring Machine Translation Post-Editing Productivity." Proceedings of AMTA 2014 Workshop on Post-editing Technology and Practice, Vancouver, pp. 60-72. 
Garcia, Ignacio. (2011). "Translating by Post-editing: Is It the Way Forward?" Machine Translation 25, no. 3, pp. 217-237. Accessed May 1, 2020, www.jstor.org/stable/41487495. https://doi.org/10.1007/s10590-011-9115-8

Guerberof, Ana. (2009a). "Productivity and Quality in MT Post-Editing." Proceedings of Machine Translation Summit XII, pp. 8-13.

Guerberof, Ana. (2009b). "Productivity and Quality in the Post-Editing of Outputs from Translation Memories and Machine Translation." The International Journal of Localisation 7(1), pp. 11-21.

Guerberof, Ana. (2013). "What Do Professional Translators Think about Post-Editing?" The Journal of Specialised Translation (19), pp. 75-95.

Hu, Ke and Patrick Cadwell. (2016). "A Comparative Study of Post-editing Guidelines."

Proceedings of the 19th Annual Conference of the European Association for Machine Translation, pp. 342-359. Accessed May 1, 2020. DOI: 10.13140/RG.2.1.2253.1446.

Jia, Y., Michael Carl and X. Wang. (2019). "How Does the Post-Editing of Neural Machine Translation Compare with From-Scratch Translation? A Product and Process Study." Journal of Specialised Translation, 31, pp. 60-86.

Katan, David. (2011). "Occupation or Profession: A Survey of the Translators' World." in Profession, identity and status: Translators and interpreters as an occupational group, edited by R. S.-S. M. Shlesinger. Amsterdam/Philadelphia: John Benjamins, pp. 65-88.

Kenny, Dorothy and Stephen Doherty. (2014). "Statistical machine translation in the translation curriculum: overcoming obstacles and empowering translators". The Interpreter and Translator Trainer, v. 8, n.2, pp. 276-294. https://doi.org/10.1080/1750399X.2014.936112

Kirchhoff, Katrin, Anne M. Turner, Amittai Axelrod, and Francisco Saavedra. (2011). "Application of Statistical Machine Translation to Public Health Information: A Feasibility Study." Journal of the American Medical Information Association 18 (4): pp. 473-478. Accessed May 1, 2020. https://doi.org/10.1136/amiajnl-2011-000176

Koponen, Maarit. (2015). "How to teach machine translation post-editing? Experiences from a post-editing course." Proceedings of 4th Workshop on Post-Editing Technology and Practice (WPTP4). Association for Machine Translation in the Americas, pp. 2-15. Accessed May 1, 2020. https://pdfs.semanticscholar.org/2321/e3f395ef5f37f7ede60bd8dbf3d3587aa8cd.pdf

Koponen, Maarit. (2016). Is post-editing worth the effort? A survey of research into post-editing and effort. Journal of Specialised Translation 25: pp. 131-148.

Krings, Hans P. (2001). Repairing texts: Empirical investigations of machine translation post-editing process. Kent, OH: The Kent State University Press.

Klubicka, Filip, Antonio Toral and Víctor M. Sánchez-Cartagena. (2018). "Quantitative fine-grained human evaluation of machine translation systems: a case study on 
English to Croatian." Machine Translation 32(3), pp. 195-215. https://doi.org/10.1007/s10590-018-9214-x

Lagoudaki, Elina. (2006). "Translation Memories Survey 2006: Users' Perceptions around TM Use." Proceeding of the 28th Conference Translation and the Computer, pp. 1516.

Läubli, Samuel and David Orrego-Carmona. (2017a). "When Google Translate Is Better than Some Human Colleagues, Those People Are No Longer Colleagues."

Proceedings of the 39th Conference Translation and the Computer, pp. 59-69.

LeBlanc, M. (2013). "Translators on Translation Memory (TM): Results of an Ethnographic Study in Three Translation Agencies and Services." Meta 59(3), pp. 537-56. https://doi.org/10.7202/1028656ar

Lommel, Arle R. and Donald A. Depalma. (2016). Europe's Leading Role in Machine Translation. Common Sense Advisory, Boston.

Meijer, Siety. (1993). "Attitudes towards Machine Translation." Language International 5, pp. 11-13.

Mellinger, C.D. (2017). "Translators and machine translation: knowledge and skills gaps in translator pedagogy." The Interpreter and Translator Trainer, pp. 280-293. https://doi.org/10.1080/1750399X.2017.1359760

Moorkens, Joss. (2017). "Under Pressure: Translation in Times of Austerity." Perspectives: Studies in Translatology 25(3), pp. 464-77. https://doi.org/10.1080/0907676X.2017.1285331

Moorkens, Joss and Sharon O'Brien. (2013). "User Attitudes to the Post-Editing Interface." Proceedings of MT Summit XIV Workshop on Post-editing Technology and Practice, pp. 19-25.

Moorkens, J. and Sharon O'Brien. (2014). "Post-Editing Evaluations: Trade-offs between Novice and Professional Participants." Proceedings of the 18th Annual Conference of the European Association for Machine Translation (EAMT 2015), pp. 75-81.

Moorkens, Joss, Sharon O'Brien, Igor A L Da Silva, Norma B. de Lima Fonseca \& Fabio Alves. (2015). "Correlations of perceived post-editing effort with measurements of actual effort". Machine Translation 29, pp. 267-284. https://doi.org/10.1007/s10590015-9175-2

Moorkens, Joss and Sharon O'Brien. (2017). "Assessing User Interface Needs of PostEditors of Machine Translation." in Human Issues in Translation Technology: The IATIS Yearbook, edited by D. Kenny. London: Routledge, pp. 109-130.

O'Brien, Sharon. (2002). "Teaching post-editing: A proposal for course content." 6th EAMT Workshop "Teaching machine translation". 14-15 November 2002, pp. 99-106.

O'Brien, Sharon. (2005). "Methodologies for Measuring the Correlations between PostEditing Effort and Machine Translatability." Machine Translation 19, pp. 37-58. https://doi.org/10.1007/s10590-005-2467-1 
O'Brien, Sharon. (2011). "Towards predicting post-editing productivity." Machine Translation, 25(3), pp. 197-215. https://doi.org/10.1007/s10590-011-9096-7

Parra Escartín, Carla and Manuel Arcedillo. (2015). "A fuzzier approach to Machine translation evaluation: A pilot study on post-editing productivity and automated metrics in commercial settings." Proceedings of the ACL 2015 Fourth Workshop on Hybrid Approaches to Translation (HyTra) 1(2010), pp. 40-45. https://aclweb.org/anthology/W/W15/W15-4107.pdf

Plitt, Mirko and François Masselot. (2010). "A Productivity Test of Statistical Machine Translation Post-Editing in a Typical Localisation Context." The Prague Bulletin of Mathematical Linguistics 93, pp. 7-16. https://doi.org/10.2478/v10108-010-0010-x

Rico Pérez, Celia and Enrique Torrejón. (2012). "Skills and profile of the new role of the translator as MT post-editor." Tradumática (10), pp. 166-178. https://doi.org/10.5565/rev/tradumatica.18

Screen, Benjamin. (2017). "Machine Translation and Welsh: Analysing Free Statistical Machine Translation for the Professional Translation of an under-Researched Language Pair." Journal of Specialized Translation 28, pp. 317-344.

Sosoni, Vilelmini and Margaret Rogers. (2013). "Translation in an Age of Austerity: From Riches to Pauper, or Not?" MTm 5, pp. 5-17.

Specia, Lucia. (2011). "Exploiting Objective Annotations for Measuring Translation PostEditing Effort." Proceedings of the European Association for Machine Translation: pp. 73-80.

Teixeira, Carlos. (2014). "Perceived vs. measured performance in the post-editing of suggestions from machine translation and translation memories." Proceedings of the Third Workshop on Post-editing Techniques and Practices (WPTP-3): The 11th Conference of the Association for Machine Translation in the Americas: Vancouver, BC Canada. AMTA, pp. 45-59.

Vieira, Lucas Nunes. (2018). "Automation Anxiety and Translators." Translation Studies 13(1), pp. 1-21. https://doi.org/10.1080/14781700.2018.1543613

Vieira, Lucas Nunes and Elisa Alonso. (2018). The Use of Machine Translation in Human Translation Workflows: Practices, Perceptions and Knowledge Exchange. Report. Institute of Translation and Interpreting. http://www/iti.org.uk/images/downloads/ITIReport-Lucas/pdf (consulted 30.10.2018). 